\title{
Weak radiative corrections to Drell-Yan process for large invariant mass of di-lepton pair
}

\author{
V.A. Zykunov* \\ Gomel State Technical University, 246746 Gomel, Belarus
}

(Dated: September 29, 2005)

\begin{abstract}
The weak radiative corrections to Drell-Yan process above the Z-peak have been studied. The compact asymptotic expression for the two heavy boson exchange - one of significant contributions to the investigated process - has been obtained. The results expand in the powers of the Sudakov electroweak logarithms. The numerical analysis in the high energy region is performed. To simulate the detector acceptance we used the standard CMS cuts. It has been ascertained that the considered radiative corrections become large at high di-lepton mass $M$ and increase the di-lepton mass distribution up to $\sim 8 \%$ and the forward-backward asymmetry up to $\sim 1.4 \%$ at LHC energies and $M \geq 1 \mathrm{TeV}$. The results can be used for the necessities of future LHC experiments.
\end{abstract}

*Electronic address: zykunov@gstu.gomel.by, Uladzimir.Zykunou@cern.ch 


\section{INTRODUCTION}

Despite the fact that the Standard Model (SM) more than twenty years keeps for oneself the status of consistent and experimentally confirmed theory, the search of New Physics (NP) manifestations is continued. The possible traces of NP can be the supersymmetry, the large extra dimensions [1] or extra neutral gauge bosons [2]. Forthcoming experiments at the collider LHC probably will shed the light on this problem.

One of powerful tool in the experiments at LHC from the point of view the NP exploration is the experimental investigation of the continuum for the Drell-Yan production of di-lepton pair, i.e. data on the cross section and the forward-backward asymmetry of the reaction

$$
p p \rightarrow \gamma, Z \rightarrow l^{+} l^{-} X
$$

at large invariant mass of di-lepton pair. The reason is the di-lepton continuum is very sensitive to any modification of SM induced by new physics. The studies of NP effects is impossible without the exact knowledge of SM predictions including higher order QCD and electroweak radiative corrections.

There is the numerous literature on this question and let us now to determine the place of our paper among this (further we will discuss only electroweak corrections since the QCD corrections are beyond the scope of the present work). So, all of electroweak corrections to (11) can be divided conventionally at three categories:

- (I) electroweak corrections induced by gauge boson self-energies,

- (II) the others QED corrections (i.e. radiative corrections induced by at least one additional photon: virtual or real),

- (III) the others Weak Radiative Corrections (WRC) (i.e. radiative corrections induced by additional heavy bosons: $Z$ or $W$ ),

The first and the second contributions are studied well (see papers on pure QED corrections 3], and QED and electroweak corrections in the $Z$-peak region and above in paper [4] and numerous papers cited there). Naturally the contribution (II) demands to consider also the diagrams with real bremsstrahlung (to cancel the infrared divergence) and therefore supposes the experimentally-motivated kinematical cuts for observed photon, all of that is under control in paper [4]. 
It is well known that (I) and (II) contributions discussed above do not contain the socalled Sudakov double logarithms (SDL) [5], i.e. the expressions which are growing with the scale of energy, and therefore they give one of the leading effect in the region of large invariant di-lepton mass. Let us specify now that (I) contribution contains only single Sudakov logarithm, and in the (II) contribution there is also double logarithms (in $Z \gamma$-boxes), but they are mutually cancelled out and reduced to single ones (see Section IV). At last, the (III) contribution contains the Sudakov double logarithms growing with di-lepton mass $M$ and, as it will be shown below, predominating in the region $M \gg m_{Z}$. Obviously, the collinear logarithms of (II) QED radiative corrections can give the contribution to compete with the SDL in investigated region. This important question has been remained undecided yet.

So, in the following we will introduce basic notations (Section II), show how to calculate the cross section corresponding to the (III) contribution diagrams (see Fig.1,b-g): the heavy vertices (Section III), the heavy boxes (Section IV) and give the numerical estimations of radiative corrections to Drell-Yan cross section and forward-backward asymmetry in the region $0.1 \mathrm{TeV} \leq M<14 \mathrm{TeV}$ (Section $\mathrm{V}$ ).

\section{NOTATIONS AND BORN CROSS SECTION OF DRELL-YAN PROCESS}

The Born cross section for the inclusive hadronic reaction $A B \rightarrow l^{+} l^{-} X$ is given in Quark Parton Model by formula

$$
d \sigma_{0}=\frac{4 \alpha^{2}}{3 S} \int d \Gamma \sum_{i, j=\gamma, Z} \operatorname{Re} \hat{D}^{i s} \hat{D}^{*}{ }^{*} \sum_{\chi=+,-} \hat{B}_{\chi} \sum_{q=u, d, s, \ldots} F_{k}^{q}\left(x_{1}, x_{2}\right) \lambda_{q_{\chi}}^{i, j} \lambda_{l \chi}^{i, j},
$$

where our notations are following (see Fig.1,a): $p_{1}$ is the 4-momentum of a first unpolarized (anti)quark with flavor $q$ and mass $m_{q} ; p_{2}$ is the 4-momentum of a second (anti)quark with flavor $q$ and mass $m_{q} ; k_{1}\left(k_{2}\right)$ is the 4-momentum of a final charged lepton $l^{+}\left(l^{-}\right)$with mass $m ; q=k_{1}+k_{2}$ is the 4-momentum of the $i$-boson with mass $m_{i}$. Invariant mass of di-lepton is $M=\sqrt{q^{2}}$. We use the standard set of Mandelstam invariants for the partonic elastic scattering $s, t, u$ :

$$
s=\left(p_{1}+p_{2}\right)^{2}, t=\left(p_{1}-k_{1}\right)^{2}, u=\left(k_{1}-p_{2}\right)^{2}
$$

and invariant $S=\left(P_{A}+P_{B}\right)^{2}$ for hadron scattering. The number $1 / 3$ is a color factor, $\alpha$ is the electromagnetic fine structure constant, the two-particle phase space element has the 
form

$$
d \Gamma=\frac{d x_{1}}{x_{1}} \frac{d x_{2}}{x_{2}} \frac{d^{3} k_{1}}{2 k_{1}^{0}} \frac{d^{3} k_{2}}{2 k_{2}^{0}} \delta\left(q-\hat{p}_{1}-\hat{p}_{2}\right),
$$

the propagator for $j$-boson has the form

$$
D^{j s}=\frac{1}{s-m_{j}^{2}+i m_{j} \Gamma_{j}},
$$

where $\Gamma_{j}$ is the $j$-boson width. The $B_{ \pm}$are the typical combinations for Drell-Yan process $B_{ \pm}=t^{2} \pm u^{2}$. The combinations of parton density functions look like

$$
F_{ \pm}^{q}\left(x_{1}, x_{2}\right)=f_{q}^{A}\left(x_{1}\right) f_{\bar{q}}^{B}\left(x_{2}\right) \pm f_{\bar{q}}^{A}\left(x_{1}\right) f_{q}^{B}\left(x_{2}\right)
$$

where $f_{q}^{H}(x)$ is the probability of finding constituent $q$ with the fraction $x$ of the hadron's momentum in hadron $H$.

The combinations of coupling constants for $f$-fermion with $i$ - (or $j$-) boson have a form

$$
\lambda_{f+}^{i, j}=v_{f}^{i} v_{f}^{j}+a_{f}^{i} a_{f}^{j}, \lambda_{f-}^{i, j}=v_{f}^{i} a_{f}^{j}+a_{f}^{i} v_{f}^{j},
$$

where

$$
v_{f}^{\gamma}=-Q_{f}, a_{f}^{\gamma}=0, v_{f}^{Z}=\frac{I_{f}^{3}-2 s_{W}^{2} Q_{f}}{2 s_{W} c_{W}}, a_{f}^{Z}=\frac{I_{f}^{3}}{2 s_{W} c_{W}},
$$

$Q_{f}$ is the charge of fermion $f, I_{f}^{3}$ is the third component of the weak isospin of fermion $f$, and $s_{W}\left(c_{W}\right)$ is the sine(cosine) of the weak mixing angle: $s_{W}=\sqrt{1-c_{W}^{2}}, c_{W}=m_{W} / m_{Z}$.

In accordance with Quark Parton Model we substitute $p_{1(2)} \rightarrow x_{1(2)} P_{A(B)}$, where $P_{A(B)}$ is the 4-momentum of initial nucleons $\mathrm{A}(\mathrm{B})$ with masses $m_{A(B)}, x_{1(2)}$ is the fraction of the nucleon $A(B)$ momentum that is carried by the incoming quarks. We have denoted this procedure by operator "hat". The first summation runs over two possible intermediate bosons: $\gamma, Z$. The third summation runs over all contributing parton configurations.

After reduction the phase space to variables $M$ and $y$ - rapidity of di-lepton (we need also the variable $\tau$ which is determined by $\tau^{2}=q^{2} / S$ ) we have a three-, two- and one-fold cross sections (yet without any experimental restrictions)

$$
\begin{gathered}
\sigma(M, y, \zeta) \equiv \frac{d^{3} \sigma}{d M d y d \zeta} \\
\sigma(M, y) \equiv \frac{d^{2} \sigma}{d M d y}=\int_{-1}^{+1} d \zeta \sigma(M, y, \zeta)
\end{gathered}
$$


and

$$
\sigma(M) \equiv \frac{d \sigma}{d M}=\int_{-\log \frac{\sqrt{S}}{M}}^{+\log \frac{\sqrt{S}}{M}} d y \int_{-1}^{+1} d \zeta \sigma(M, y, \zeta),
$$

then the Born cross section looks like

$$
\sigma_{0}(M, y, \zeta)=\frac{2 \pi \alpha^{2}}{3 S M} \operatorname{Re} \sum_{i, j=\gamma, Z} D^{i s} D^{j s^{*}} \sum_{\chi=+,-}\left(t^{2}+\chi u^{2}\right) \lambda_{l}^{i, j} \sum_{q=u, d, s, \ldots} F_{\chi}^{q}\left(x_{+}, x_{-}\right) \lambda_{q \chi}^{i, j},
$$

where the invariants $s, t$ and $u$ mean

$$
s=M^{2}, t=-\frac{1}{2} s(1-\zeta), u=-\frac{1}{2} s(1+\zeta),
$$

$\zeta$ is cosine of angle $\theta$ between $\vec{p}_{1}$ and $\vec{k}_{1}$ in center mass system of hadrons $(\zeta=\cos \theta)$ and the arguments of parton distribution functions in (12) have a form

$$
x_{ \pm}=\tau e^{ \pm y}
$$

Integrating over $\zeta$ we get much more simple expression

$$
\sigma_{0}(M, y)=\frac{8 \pi \alpha^{2} M^{3}}{9 S} \operatorname{Re} \sum_{i, j=\gamma, Z} D^{i s} D^{j s^{*}} \lambda_{l+}^{i, j} \sum_{q=u, d, s, \ldots} F_{+}^{q}\left(x_{+}, x_{-}\right) \lambda_{q+}^{i, j} .
$$

\section{HEAVY VERTICES}

To construct the contribution to radiative corrections of Heavy Vertices (HV), see diagrams (b-e) in Fig.1, we used the t'Hooft-Feynman gauge and on-mass renormalization scheme which uses $\alpha, m_{W}, m_{Z}$, Higgs boson mass $m_{H}$ and the fermion masses as independent parameters. Appropriate (for ultrarelativistic limit we are interested) results can be taken from Ref. [6], where renormalized gauge boson fermion vertices for on shell fermions have been obtained. These results are presented as the form factor set to the Born vertices, so we can easily use they to construct the cross section: all that we need is to replace the coupling constants in Born vertex to the corresponding form factors:

$$
v_{f}^{j} \rightarrow F_{V}^{j f}, a_{f}^{j} \rightarrow F_{A}^{j f}
$$

Then the cross section of heavy vertices contribution looks like

$$
\begin{aligned}
\sigma_{V}(M, y, \zeta)=\frac{4 \pi \alpha^{2}}{3 S M} & \operatorname{Re} \sum_{i, j=\gamma, Z} D^{i s} D^{j s^{*}} \sum_{\chi=+,-}\left(t^{2}+\chi u^{2}\right) \times \\
& \times \sum_{q=u, d, s, \ldots} F_{\chi}^{q}\left(x_{+}, x_{-}\right)\left(\lambda_{q \chi}^{F i, j} \lambda_{l \chi}^{i, j}+\lambda_{q \chi}^{i, j} \lambda_{l \chi}^{F i, j}\right),
\end{aligned}
$$


where combinations of couplings constants and form factors are

$$
\lambda_{f}^{F_{+}^{i, j}}=F_{V}^{i f} v_{f}^{j}+F_{A}^{i f} a_{f}^{j}, \quad \lambda_{f}^{F_{-}^{i, j}}=F_{V}^{i f} a_{f}^{j}+F_{A}^{i f} v_{f}^{j}, \quad(f=q, l) .
$$

Electroweak form factors $F_{V, A}^{i f}$ in ultrarelativistic limit have a form (there is the perfect coincidence with the formulas of Appendix C of Ref.[7]):

$$
\begin{aligned}
& F_{V}^{\gamma l}=\frac{\alpha v_{l}^{\gamma}}{4 \pi}\left[\left(\left(v_{l}^{Z}\right)^{2}+\left(a_{l}^{Z}\right)^{2}\right) \Lambda_{2}\left(m_{Z}\right)+\frac{3}{4 s_{W}^{2}} \Lambda_{3}\left(m_{W}\right)\right], \\
& F_{A}^{\gamma l}=\frac{\alpha v_{l}^{\gamma}}{4 \pi}\left[2 v_{l}^{Z} a_{l}^{Z} \Lambda_{2}\left(m_{Z}\right)+\frac{3}{4 s_{W}^{2}} \Lambda_{3}\left(m_{W}\right)\right], \\
& F_{V}^{\gamma d}=\frac{\alpha v_{d}^{\gamma}}{4 \pi}\left[\left(\left(v_{d}^{Z}\right)^{2}+\left(a_{d}^{Z}\right)^{2}\right) \Lambda_{2}\left(m_{Z}\right)-\frac{1}{2 s_{W}^{2}} \Lambda_{2}\left(m_{W}\right)+\frac{9}{4 s_{W}^{2}} \Lambda_{3}\left(m_{W}\right)\right] \\
& F_{A}^{\gamma d}=\frac{\alpha v_{d}^{\gamma}}{4 \pi}\left[2 v_{d}^{Z} a_{d}^{Z} \Lambda_{2}\left(m_{Z}\right)-\frac{1}{2 s_{W}^{2}} \Lambda_{2}\left(m_{W}\right)+\frac{9}{4 s_{W}^{2}} \Lambda_{3}\left(m_{W}\right)\right] \\
& F_{V}^{\gamma u}=\frac{\alpha v_{u}^{\gamma}}{4 \pi}\left[\left(\left(v_{u}^{Z}\right)^{2}+\left(a_{u}^{Z}\right)^{2}\right) \Lambda_{2}\left(m_{Z}\right)-\frac{1}{8 s_{W}^{2}} \Lambda_{2}\left(m_{W}\right)+\frac{9}{8 s_{W}^{2}} \Lambda_{3}\left(m_{W}\right)\right] \\
& F_{A}^{\gamma u}=\frac{\alpha v_{u}^{\gamma}}{4 \pi}\left[2 v_{u}^{Z} a_{u}^{Z} \Lambda_{2}\left(m_{Z}\right)-\frac{1}{8 s_{W}^{2}} \Lambda_{2}\left(m_{W}\right)+\frac{9}{8 s_{W}^{2}} \Lambda_{3}\left(m_{W}\right)\right] . \\
& F_{V}^{Z l}=\frac{\alpha}{4 \pi}\left[v_{l}^{Z}\left(\left(v_{l}^{Z}\right)^{2}+3\left(a_{l}^{Z}\right)^{2}\right) \Lambda_{2}\left(m_{Z}\right)+\frac{1}{8 s_{W}^{3} c_{W}} \Lambda_{2}\left(m_{W}\right)-\frac{3 c_{W}}{4 s_{W}^{3}} \Lambda_{3}\left(m_{W}\right)\right], \\
& F_{A}^{Z l}=\frac{\alpha}{4 \pi}\left[a_{l}^{Z}\left(3\left(v_{l}^{Z}\right)^{2}+\left(a_{l}^{Z}\right)^{2}\right) \Lambda_{2}\left(m_{Z}\right)+\frac{1}{8 s_{W}^{3} c_{W}} \Lambda_{2}\left(m_{W}\right)-\frac{3 c_{W}}{4 s_{W}^{3}} \Lambda_{3}\left(m_{W}\right)\right], \\
& F_{V}^{Z d}=\frac{\alpha}{4 \pi}\left[v_{d}^{Z}\left(\left(v_{d}^{Z}\right)^{2}+3\left(a_{d}^{Z}\right)^{2}\right) \Lambda_{2}\left(m_{Z}\right)+\frac{1-2 Q_{u} s_{W}^{2}}{8 s_{W}^{3} c_{W}} \Lambda_{2}\left(m_{W}\right)-\frac{3 c_{W}}{4 s_{W}^{3}} \Lambda_{3}\left(m_{W}\right)\right] \\
& F_{A}^{Z d}=\frac{\alpha}{4 \pi}\left[a_{d}^{Z}\left(3\left(v_{d}^{Z}\right)^{2}+\left(a_{d}^{Z}\right)^{2}\right) \Lambda_{2}\left(m_{Z}\right)+\frac{1-2 Q_{u} s_{W}^{2}}{8 s_{W}^{3} c_{W}} \Lambda_{2}\left(m_{W}\right)-\frac{3 c_{W}}{4 s_{W}^{3}} \Lambda_{3}\left(m_{W}\right)\right] \\
& F_{V}^{Z u}=\frac{\alpha}{4 \pi}\left[v_{u}^{Z}\left(\left(v_{u}^{Z}\right)^{2}+3\left(a_{u}^{Z}\right)^{2}\right) \Lambda_{2}\left(m_{Z}\right)-\frac{1+2 Q_{d} s_{W}^{2}}{8 s_{W}^{3} c_{W}} \Lambda_{2}\left(m_{W}\right)+\frac{3 c_{W}}{4 s_{W}^{3}} \Lambda_{3}\left(m_{W}\right)\right] \\
& F_{A}^{Z u}=\frac{\alpha}{4 \pi}\left[a_{u}^{Z}\left(3\left(v_{u}^{Z}\right)^{2}+\left(a_{u}^{Z}\right)^{2}\right) \Lambda_{2}\left(m_{Z}\right)-\frac{1+2 Q_{d} s_{W}^{2}}{8 s_{W}^{3} c_{W}} \Lambda_{2}\left(m_{W}\right)+\frac{3 c_{W}}{4 s_{W}^{3}} \Lambda_{3}\left(m_{W}\right)\right] .
\end{aligned}
$$

Here it is necessary to say that in on-mass renormalization scheme the self-energies of $u$-quarks diagrams give also non-zero contribution to the cross section. However, the results for $u$-self-energy are factorized absolutely in the manner of vertices. It gives possibility 
to sum both of contributions in general formula at that the $u$-self-energy contribution is completely cancelled with corresponding terms of heavy vertices. So it can say that formula (17) is general cross section induced by both heavy vertices and self-energy of $u$-quarks.

Now we give the real part of expressions for functions $\Lambda_{2,3}\left(m_{i}\right)$ that are provided a objective accuracy in large di-lepton mass region (see Introduction):

$$
\Lambda_{2}\left(m_{i}\right)=\frac{\pi^{2}}{3}-\frac{7}{2}-3 l_{i, s}-l_{i, s}^{2}, \quad \Lambda_{3}\left(m_{i}\right)=\frac{5}{6}-\frac{1}{3} l_{i, s} .
$$

At last the Sudakov logarithm that we denote as $l_{i, x}$ is the leading term in the region we are interested

$$
l_{i, x}=\log \frac{m_{i}^{2}}{|x|} \quad(i=Z, W ; \quad x=s, t, u) .
$$

\section{HEAVY BOXES}

The calculation of two heavy boson (box) contribution is more complicate procedure since it demands the integration of 4-point functions with complex masses in unlimited from above kinematical region of invariants. It have been pointed on the difficulties of such procedure as far back as in pioneer paper [8]. Fortunately there is a way to avoid many of troubles with the integration all of terms in box contribution. Let us expound it.

First of all we construct the $Z Z$-box cross section for $q \bar{q} \rightarrow l^{+} l^{-}$(see diagrams in Fig.1,f-g) using the standard Feynman rules:

$$
d \sigma_{Z Z}=-\frac{4 \alpha^{3}}{\pi s} \delta\left(q-p_{1}-p_{2}\right) \frac{d^{3} k_{1}}{2 k_{1}^{0}} \frac{d^{3} k_{2}}{2 k_{2}^{0}} \operatorname{Re} \frac{i}{(2 \pi)^{2}} \int d^{4} k \sum_{k=\gamma, Z} D^{k s^{*}}\left(D^{Z Z}+C^{Z Z}\right),
$$

here $D^{Z Z}\left(C^{Z Z}\right)$ is contribution of direct (crossed) diagram.

Neglecting of fermion masses and polarization of particles we present the direct contribution in the form:

$$
D^{Z Z}=\Pi_{Z Z}^{D} \operatorname{Tr}\left[\gamma^{\alpha} \hat{p}_{2} \gamma_{\mu}\left(\hat{p}_{1}-\hat{k}\right) \gamma_{\nu} \rho_{q}^{Z Z, k}\left(p_{1}\right)\right] \operatorname{Tr}\left[\gamma_{\alpha} \hat{k}_{2} \gamma^{\mu}\left(\hat{k}-\hat{k}_{1}\right) \gamma^{\nu} \rho_{l}^{Z Z, k}\left(k_{1}\right)\right],
$$

where integrand of 4-point scalar function looks like

$$
\Pi_{Z Z}^{D}=\frac{1}{\left((q-k)^{2}-m_{Z}^{2}\right)\left(k^{2}-m_{Z}^{2}\right)\left(k^{2}-2 k_{1} k\right)\left(k^{2}-2 p_{1} k\right)} .
$$

Combinations of the density matrices $\rho(p)$ and the coupling constants can be reduced to production of $\lambda$-factors (see formulas (77)) and $\hat{p} \equiv \gamma_{\mu} p^{\mu}$ (here $p=p_{1}, p_{2}, k_{1}, k_{2}$ or $k$ )

$$
\rho_{f}^{Z Z, k}(p)=\left(v_{f}^{Z Z}-a_{f}^{Z Z} \gamma_{5}\right) \rho(p)\left(v_{f}^{k}+a_{f}^{k} \gamma_{5}\right)=\frac{1}{2}\left(\lambda_{f}^{Z Z, k}-\lambda_{f}^{Z Z, k} \gamma_{5}\right) \hat{p},
$$




$$
v_{f}^{Z Z}=\left(v_{f}^{Z}\right)^{2}+\left(a_{f}^{Z}\right)^{2}, a_{f}^{Z Z}=2 v_{f}^{Z} a_{f}^{Z} .
$$

To extract the part of cross section which predominates in region $|x| \gg m_{Z}^{2}$ (see $x$ in formula (22) ) we should make equivalent transformation of the cross section based on the close connection of infrared divergency cross section terms and the Sudakov leading-log terms:

$$
D^{Z Z}=\left(\left.D^{Z Z}\right|_{k \rightarrow 0}+\left.D^{Z Z}\right|_{k \rightarrow q}\right)+\left(D^{Z Z}-\left.D^{Z Z}\right|_{k \rightarrow 0}-\left.D^{Z Z}\right|_{k \rightarrow q}\right)=D_{1}^{Z Z}+D_{2}^{Z Z}
$$

The integral over $k$ of first term is

$$
\begin{aligned}
\frac{i}{(2 \pi)^{2}} \int d^{4} k D_{1}^{Z Z}=\frac{4 t B^{Z Z, k}}{q^{2}-m_{Z}^{2}} \times \frac{i}{(2 \pi)^{2}} \int d^{4} k \frac{1}{k^{2}-m_{Z}^{2}} & {\left[\frac{1}{\left(k^{2}-2 k_{1} k\right)\left(k^{2}-2 p_{1} k\right)}+\right.} \\
& \left.+\frac{1}{\left(k^{2}-2 k_{2} k\right)\left(k^{2}-2 p_{2} k\right)}\right]
\end{aligned}
$$

here we used trivial correlation typical for Born cross section

$$
B^{Z Z, k}=\frac{1}{2} \operatorname{Tr}\left[\gamma^{\alpha} \hat{p}_{2} \gamma_{\mu} \rho_{q}^{Z Z, k}\left(p_{1}\right)\right] \operatorname{Tr}\left[\gamma_{\alpha} \hat{k}_{2} \gamma^{\mu} \rho_{l}^{Z Z, k}\left(k_{1}\right)\right] \approx b_{+}^{Z Z, k} t^{2}+b_{-}^{Z Z, k} u^{2},
$$

where

$$
b_{ \pm}^{n, k}=\lambda_{q_{+}}^{n, k} \lambda_{l+}^{n, k} \pm \lambda_{q_{-}}^{n, k} \lambda_{l-}^{n, k}
$$

Then we integrate over $k$ the one of terms in (29) using standard method of paper [8] (other term is integrating similarly):

$$
\begin{aligned}
& \frac{i}{(2 \pi)^{2}} \int \frac{d^{4} k}{\left(k^{2}-m_{Z}^{2}\right)\left(k^{2}-2 k_{2} k\right)\left(k^{2}-2 p_{2} k\right)}=\frac{1}{4} \int_{0}^{1} d x \int_{0}^{x} \frac{d y}{m_{Z}^{2}(x-y)+\left(p_{2}(1-x)+k_{2} y\right)^{2}}= \\
& =\frac{1}{4} \int_{0}^{1} d x \frac{1}{t(x-1)-m_{Z}^{2}} \log \frac{t(x-1)}{m_{Z}^{2}}=-\frac{1}{4 t}\left(\frac{\pi^{2}}{3}+\frac{1}{2} \log ^{2} \frac{-t-m_{Z}^{2}}{m_{Z}^{2}}+\operatorname{Li}_{2} \frac{m_{Z}^{2}}{m_{Z}^{2}+t}\right),
\end{aligned}
$$

here $\mathrm{Li}_{2}$ denotes the Spence dilogarithm. Saving in the calculation the terms which are proportional to the second $\left(\sim l_{i, x}^{2}\right)$, first $\left(\sim l_{i, x}^{1}\right)$ and zero $\left(\sim l_{i, x}^{0}\right)$ power of Sudakov logarithms and thereby neglecting the terms which are inessential in the region of large di-lepton mass $|x| \gg m_{Z}^{2}$ we get the asymptotic expression

$$
\frac{i}{(2 \pi)^{2}} \int d^{4} k D_{1}^{Z Z} \approx-\frac{2}{s} B^{Z Z, k}\left(\frac{\pi^{2}}{3}+\frac{1}{2} l_{Z, t}^{2}\right) .
$$

Surely in these (and subsequent) expressions we can retain only leading $\sim l_{i, x}^{2}$ term how it had been done in [9]. Naturally in such approximation our results absolutely coincide 
with the results of this paper (see formula (8) in paper [9]). However we are in position to save the first and zero power of expansion leading-log parameter and we will seize this opportunity to improve the accuracy of radiative correction estimation (one more remark we retain the $l_{i, x}^{1}$ and $l_{i, x}^{0}$ also in heavy vertices part, of course).

The asymptotic at $|x| \gg m_{Z}^{2}$ expression for the second term of formula (28) can be presented as

$$
\left.D_{2}^{Z Z} \approx D^{Z Z}\right|_{\Pi_{Z Z}^{D} \rightarrow \Pi_{\gamma \gamma}^{D}}-4 \Pi_{\gamma \gamma}^{D} \frac{(q-k)^{2}+k^{2}}{q^{2}} t B^{Z Z, k}
$$

Reducing the vectorial and tensor integrals to scalar ones we get

$$
\begin{aligned}
\frac{i}{(2 \pi)^{2}} \int d^{4} k D_{2}^{Z Z} \approx & -2 b_{-}^{Z Z, k}\left[\left(G_{0}^{m}+G_{0}^{M}\right)\left(t^{2}+u^{2}\right)-2\left(R-N_{0}\right) u-X_{0} t \frac{t^{2}+u^{2}}{s}\right]- \\
& -4 b_{+}^{Z Z, k} t^{2}\left[G_{0}^{m}+G_{0}^{M}-X_{0} \frac{t}{s}\right]
\end{aligned}
$$

where scalar integrals are

$$
\begin{aligned}
G_{0}^{m} & =\frac{i}{(2 \pi)^{2}} \int \frac{d^{4} k}{\left(k^{2}-2 k_{1} k\right) k^{2}(k-q)^{2}}=-\frac{1}{8 q^{2}}\left(\log ^{2} \frac{q^{2}}{m^{2}}+\frac{\pi^{2}}{3}\right), \\
G_{0}^{M} & =\frac{i}{(2 \pi)^{2}} \int \frac{d^{4} k}{\left(k^{2}-2 p_{1} k\right) k^{2}(k-q)^{2}}=-\frac{1}{8 q^{2}}\left(\log ^{2} \frac{q^{2}}{m_{q}^{2}}+\frac{\pi^{2}}{3}\right), \\
R & =\frac{i}{(2 \pi)^{2}} \int \frac{d^{4} k}{k^{2}(k-q)^{2}}=\frac{1}{4}\left(\log \frac{q^{2}}{L}-1\right), \\
N_{0} & =\frac{i}{(2 \pi)^{2}} \int \frac{d^{4} k}{\left(k^{2}-2 k_{1} k\right)\left(k^{2}-2 p_{1} k\right)}=\frac{1}{4}\left(\log \frac{m^{2}}{L}-1-\log \frac{m}{m_{q}}+\log \frac{\left|2 k_{1} p_{1}\right|}{m_{q}}\right), \\
X_{0} & =\frac{i}{(2 \pi)^{2}} \int d^{4} k \frac{2 k(q-k)}{\left(k^{2}-2 k_{1} k\right)\left(k^{2}-2 p_{1} k\right) k^{2}(k-q)^{2}}= \\
& =-\frac{1}{8 p_{1} k_{1}}\left(\frac{1}{2} \log \frac{\left|2 p_{1} k_{1}\right| m^{2}}{q^{4}} \log \frac{\left|2 p_{1} k_{1}\right|}{m^{2}}+\frac{1}{2} \log \frac{\left|2 p_{1} k_{1}\right| m_{q}^{2}}{q^{4}} \log \frac{\left|2 p_{1} k_{1}\right|}{m_{q}^{2}}-\frac{\pi^{2}}{3}\right) .
\end{aligned}
$$

These integrals have been calculated analogously the corresponding ones in paper of J.Kahane [10] (with the important distinctions due to different reaction channel: $t$-channel in [10], and $s$-channel in this paper). The expressions (36) contain two sort of parameters: masses of fermions and $L$ - parameter which regulates the ultraviolet divergence, both of them are completely cancelled out in final expression

$$
\frac{i}{(2 \pi)^{2}} \int d^{4} k D_{2}^{Z Z} \approx b_{-}^{Z Z, k} l_{s} u+\left(b_{-}^{Z Z, k}\left(t^{2}+u^{2}\right)+2 b_{+}^{Z Z, k} t^{2}\right) \frac{1}{2 s} l_{s}^{2}, \quad l_{s}=\log \frac{s}{|t|} .
$$


The contribution of crossed part (Fig.1,g) to cross section can be calculated analogously to direct one. Besides that there is interesting symmetry between the direct and crossed parts (see, for example, [4]), namely

$$
C^{Z Z}=-\left.D^{Z Z}\right|_{t \leftrightarrow u} ^{b_{+}^{Z Z, k} \leftrightarrow b_{-}^{Z Z, k}} .
$$

Let us now present the total final expression for contribution of $Z Z$-box to cross section of Drell-Yan process at large invariant di-lepton mass:

$$
\begin{aligned}
\sigma_{Z Z}(M, y, \zeta)=\frac{2 \alpha^{3}}{3 S M} \operatorname{Re} \sum_{k=\gamma, Z} D^{k s^{*}} \times \\
\times \sum_{q=u, d, s, \ldots}\left[f_{q}^{A}\left(x_{+}\right) f_{\bar{q}}^{B}\left(x_{-}\right)\left(\delta^{Z Z, k}\left(t, u, b_{+}, b_{-}\right)-\delta^{Z Z, k}\left(u, t, b_{-}, b_{+}\right)\right)+\right. \\
\left.\quad+f_{\bar{q}}^{A}\left(x_{+}\right) f_{q}^{B}\left(x_{-}\right)\left(\delta^{Z Z, k}\left(u, t, b_{+}, b_{-}\right)-\delta^{Z Z, k}\left(t, u, b_{-}, b_{+}\right)\right)\right]
\end{aligned}
$$

where

$$
\delta^{Z Z, k}\left(t, u, b_{+}, b_{-}\right)=\frac{B^{Z Z, k}}{s}\left(\frac{2 \pi^{2}}{3}+l_{Z, t}^{2}\right)-b_{-}^{Z Z, k} u l_{s}-\left(b_{-}^{Z Z, k}\left(t^{2}+u^{2}\right)+2 b_{+}^{Z Z, k} t^{2}\right) \frac{l_{s}^{2}}{2 s} .
$$

Integration over $\zeta$ the formula (40) gives

$$
\begin{aligned}
\int_{-1}^{+1} \delta^{Z Z, k}\left(t, u, b_{+}, b_{-}\right) & d \zeta=\int_{-1}^{+1} \delta^{Z Z, k}\left(u, t, b_{+}, b_{-}\right) d \zeta= \\
= & \frac{s}{9}\left(12 \lambda_{q_{+}}^{Z Z, k} \lambda_{l_{+}}^{Z Z, k}\left(\frac{2 \pi^{2}}{3}+l_{Z, s}^{2}\right)+\left(22 b_{-}^{Z Z, k}+4 b_{+}^{Z Z, k}\right) l_{Z, s}+27 b_{-}^{Z Z, k}\right)(41
\end{aligned}
$$

(we will use this for the construction of twofold cross sections $\sigma_{Z Z, W W}(M, y)$ ).

To obtain the corresponding $W W$-box contribution to Drell-Yan cross section using the expressions (39, 40) one should: 1) to do the trivial substitution in all of indices of coupling constants and boson masses $Z \rightarrow W, 2$ ) to take into consideration that some parton diagrams are forbidden by the charge conservation law (direct $W W$-box: $d \bar{d} \rightarrow l^{+} l^{-}$and $\bar{u} u \rightarrow l^{+} l^{-}$; crossed $W W$-box: $u \bar{u} \rightarrow l^{+} l^{-}$and $\bar{d} d \rightarrow l^{+} l^{-}$). This second feature of $W W$-boxes explains the fact of domination of $W W$-contribution to Drell-Yan cross section in comparison with $Z Z$-contribution (see below in numerical analysis). Point is that the leading term of $Z Z$ contribution is proportional to difference

$$
\delta^{Z Z, k}\left(t, u, b_{+}, b_{-}\right)-\delta^{Z Z, k}\left(u, t, b_{-}, b_{+}\right) \sim l_{Z, t}^{2}-l_{Z, u}^{2}=\log \frac{u}{t}\left(l_{Z, t}^{1}+l_{Z, u}^{1}\right),
$$

i.e. leading terms of $Z Z$-box contribution $\sim l_{Z, x}^{1}$, whereas the leading parts of $W W$-cross section do not contain the difference (42) and are proportional to $l_{W, x}^{2}$. Let us remark here 
that the factorization property (42) is absent in heavy vertex part and takes place for infrared finite part of $\gamma Z$-box contribution, which one can easily obtain by full analogy with $Z Z$-box using

$$
\delta^{\gamma Z, k}\left(t, u, b_{+}, b_{-}\right)=\frac{B^{\gamma Z, k}}{s}\left(\frac{2 \pi^{2}}{3}+l_{Z, t}^{2}\right)-2 b_{-}^{\gamma Z, k} u l_{s}-\left(b_{-}^{\gamma Z, k}\left(t^{2}+u^{2}\right)+2 b_{+}^{\gamma Z, k} t^{2}\right) \frac{l_{s}^{2}}{s} .
$$

\section{DISCUSSION OF NUMERICAL RESULTS}

In the following the scale of weak radiative corrections and their effect on the observables of the Drell-Yan processes will be discussed. We used the following standard set of electroweak parameters: $\alpha=1 / 137.036, m_{W}=80.451 \mathrm{GeV}, m_{Z}=91.1875 \mathrm{GeV}$ [1]], the energy of LHC $\sqrt{S}=14 \mathrm{TeV}$ and the CTEQ6 set of unpolarized parton distribution functions [12] (with the choice $Q^{2}=M^{2}$ ).

Let us study the common properties of WRC without taking into consideration the some experimental cuts. For this, firstly, we present in Fig.2 the numerical results for the relative corrections

$$
\delta_{a}=\frac{\sigma_{a}(M, y, \zeta)}{\sigma_{0}(M, y, \zeta)}
$$

as a functions of $M$ for the rapidity $y=0$ and the different values of $\zeta:-0.9,0,0.9$. Index "a" in all numerical analisys means

$$
a=Z Z, Z \gamma, W W, H V
$$

It could be seen in these figures the predominance of $H V$-and $W W$-contributions which has been already discussed above. The $W W$-contribution is small only near the point $y=0, \zeta=0$, where $x_{+}=x_{-}$and there is the logarithmic cancellation similar to (42). Secondly, in Fig.3 the relative corrections

$$
\Delta_{a}=\frac{\sigma_{a}(M, y)}{\sigma_{0}(M, y)},
$$

as a functions of $M$ for different values of $y:-3,-1,0,1,3$ are presented. The main features of the behavior of these corrections in general the same as for the $\delta_{a}$ : increasing with the $M$ and predominance of $W W$ and $H V$ contributions.

In Fig. 4 we show the cross sections $\sigma(M)$ as a functions of $M$ in the region $1 \mathrm{TeV} \leq$ $M \leq 2 \mathrm{TeV}$ for different integrations over $\zeta$ and di-lepton rapidity: without experimental 
restrictions (curves denoted by 'off') and with experimental restriction conditions on detected lepton angle $-\zeta^{*} \leq \zeta \leq \zeta^{*}$ and on rapidity $|y(l)| \leq y(l)^{*}$ (curves denoted by 'on').

For CMS detector the cut values of $\zeta^{*}$ and $y(l)^{*}$ are determined as

$$
y(l)^{*}=-\log \tan \frac{\theta^{*}}{2}=2.4, \zeta^{*}=\cos \theta^{*} \approx 0.9837 .
$$

Then the expression (11) taking into consideration the experimental restrictions (47) can be modified to

$$
\sigma(M)=\int_{-\log \frac{\sqrt{S}}{M}}^{+\log \frac{\sqrt{S}}{M}} d y \int_{-\zeta^{*}}^{\zeta^{*}} d \zeta \sigma(M, y, \zeta) \theta\left(\cos \alpha+\zeta^{*}\right) \theta\left(-\cos \alpha+\zeta^{*}\right)
$$

where $\alpha$ is the scattering angle of the lepton with the 4 -momenta $k_{2}\left(\alpha=\vec{p}_{1}, \vec{k}_{2}\right)$ in center mass system of hadrons. This angle has a non-trivial relation with $\theta$ and $y$ :

$$
\alpha=\pi-\arccos \frac{\cos \theta-f}{\sqrt{1+f^{2}-2 f \cos \theta}}-\arcsin \frac{f \sin \theta}{\sqrt{1+f^{2}-2 f \cos \theta}}, f=\frac{e^{2 y}-1}{e^{2 y}+1} .
$$

Let us remark here that the second standard CMS restriction $p_{T}(l) \geq 20 \mathrm{GeV}$ in the region of large $M$ considered here and at (47) is satisfied completely and automatically. It can be seen in Fig.5. where all of experimental restrictions of CMS for $M=1 \mathrm{TeV}$ are shown.

At last, in Fig.6 we present the relative corrections

$$
d_{a}=\frac{\sigma_{a}(M)}{\sigma_{0}(M)}
$$

as a functions of $M$ for different integration over $\zeta$ and di-lepton rapidity: with (and without) experimental restrictions of CMS. It can be seen the very weak dependence of WRC on the cuts, only in the $W W$-case there is the noticeable effect. As to total WRC effect, the $\delta_{\text {total }}$ correction has slow growth in the beginning, and for $M>2.2 \mathrm{TeV}$ is approximately constant $\delta_{\text {total }} \approx 0.82$.

Now let us discuss the forward-backward asymmetry $A_{F B}$ and influence of the WRC on that observable. We will define the $A_{F B}$ as

$$
A_{F B}=\frac{\sigma_{F}(M)-\sigma_{B}(M)}{\sigma_{F}(M)+\sigma_{B}(M)}
$$

where $\sigma_{F(B)}(M)$ are the forward (backward) cross sections corresponding to the condition $\cos \theta_{l}^{*}>0\left(\cos \theta_{l}^{*}<0\right)$. Here $\theta_{l}^{*}$ is the scattering angle of lepton in the di-lepton rest frame. For the Born kinematics the expression

$$
\cos \theta_{l}^{*}= \pm \frac{1}{2}\left(e^{y}-e^{-y}\right)^{2} \frac{\sin \theta \sin \alpha}{\sin ^{2}(\theta+\alpha)}(\cos \alpha-\cos \theta)
$$


has been obtained easily, where the factor \pm 1 at the last formula is +1 for $y>0$ and -1 for $y<0$ (at $y=0$ this factor loses the sense as $\left.\cos \theta_{l}^{*}\right|_{y=0}=0$ ).

In Fig.7 it can be seen the Born and radiatively corrected forward-backward asymmetries depending on $M$. Radiative effect is significant at $M>1 \mathrm{TeV}$ and maximal at $M \sim 4 \mathrm{TeV}$. The behavior of the difference between Born and radiatively corrected asymmetries

$$
\delta_{A}=A_{F B}^{a}-A_{F B}^{0}
$$

corresponding to $Z Z, Z \gamma, W W, H V$-contributions is shown in Fig.8. The same way as for the cross sections the $W W, H V$-contributions are most significant, and the total effect is rather large at $1.5 \mathrm{TeV}<M<7 \mathrm{TeV}$, so for $M=4.2 \mathrm{TeV}$ the correction $\delta_{A}^{\text {total }} \approx 0.014$.

In the end of this paragraph we should say that the importance of investigated problem demands to cross-check the presented results with the obtained earlier, for example in Ref. [4]. The approaches in both calculations are not quite similar. Although for the calculation of the Heavy Vertex part here and in Ref. [4] the same form factors were used, but one of not simple part - the heavy boxes - was calculated in different ways: via the direct 4-point functions integration in [4], and using asymptotic property of heavy vertieces expressed through the Sudakov logarithms powers in present paper. Unfortunately, a direct comparison between our numerical results is not possible, as in Ref. [4] the effective Born approximation was used at the constructions of graphs. We do not use the effective Born approximation as long as the main subject of our investigation is the region of large invariant mass where this approach is illegal.

\section{CONCLUSIONS}

The weak radiative corrections to Drell-Yan process for large invariant mass of di-lepton pair have been studied. The results are the compact asymptotic expressions, they expand in the powers of the Sudakov electroweak logarithms. The numerical analysis in the high energy region is performed using the standard CMS cuts. It has been ascertained that the considered radiative corrections become large at high di-lepton mass and increase the cross section and forward-backward asymmetry. All of relative corrections to cross sections grow with the $M$ and their large scale does not permit to neglect this part of the radiative correction procedure in the future experiments at LHC directed on NP exploration in Drell- 
Yan process. 


\section{ACKNOWLEDGMENTS}

I would like to thank V.Mossolov, S.Shmatov and N.Shumeiko for the stimulating discussions. I am grateful to CERN (CMS Group), where part of this work was carried out, for its warm hospitality.

[1] N. Arkani-Hamed et.al, Phys. Lett. B 429, 263 (1998) hep-ph/9803315; I. Antoniadis et.al, Phys. Lett B 436, 257 (1998) hep-ph/9804398; L. Randall and R. Sundrum, Phys. Rev. Lett. 83, 3370 (1999) hep-ph/9905221, Phys. Rev. Lett. 83, 4690 (1999) hep-th/9906064; C. Kokorelis, Nucl. Phys. 677 (2004) 115 hep-th/0207234;

[2] A. Leike, Phys. Rep. 317, 143 (1999), hep-ph/9805494 T.G. Rizzo. Extended Gauge Sector at Future Colliders: Report on the New Gauge Boson Subgroup in Proc. of 1996 DPF/DPB Summer Study on New Directions for High Energy Physics-Snowmass96, Snowmass, CO, 25 June - 12 July, 1996, hep-ph/9612440,

[3] V. Mosolov and N. Shumeiko, Nucl.Phus. B 186, 394 (1981), A. Soroko and N. Shumeiko, Yad. Fiz 52, 514 (1990)

[4] U. Baur et al., Phys. Rev. D 65: 033007, (2002)

[5] V. Sudakov, Sov. Phys. JETP 3, 65 (1956)

[6] M. Böhm et al., Fortschr. Phys. 34, 687 (1986)

[7] W. Hollik, Fortschr. Phys. 38, 165 (1990)

[8] G.'t Hooft and M. Veltman, Nucl. Phys. B 153, 365 (1979)

[9] P. Ciafaloni and D. Comelli, Phys. Lett. B 446, 278 (1999)

[10] J. Kahane, Phys. Rev. B 135, 975 (1964)

[11] Particle Data Group, Phys. Lett. B 592, 31 (2004)

[12] J. Pumplin et al., JHEP 0207:012 (2002), hep-ph/0201195 


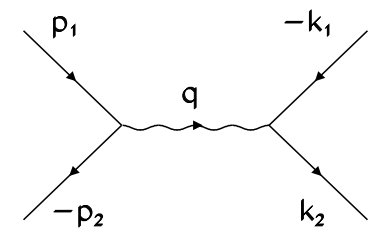

a)

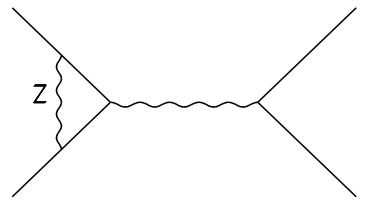

b)

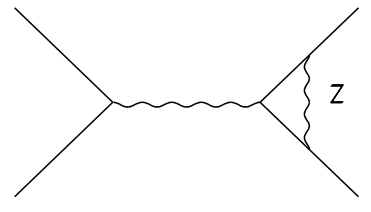

c)

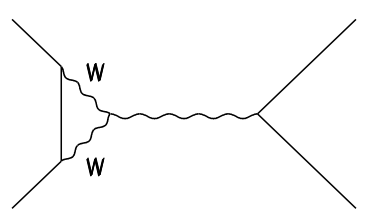

d)

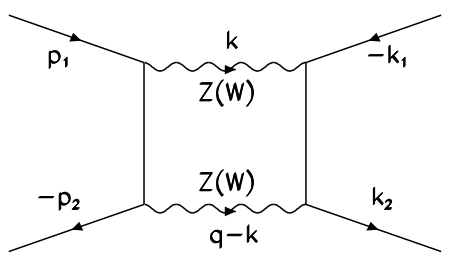

f)

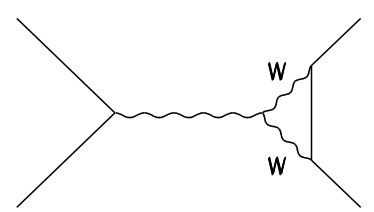

e)

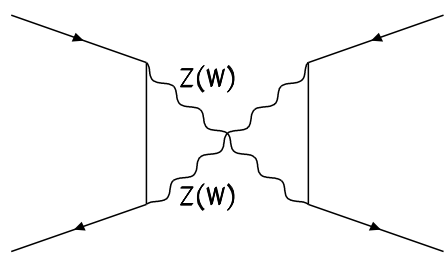

g)

FIG. 1: Feynman graphs for Born (a) and one-loop (b-g) diagrams with additional virtual heavy bosons. Unsigned helix lines mean $\gamma$ or $Z$. 

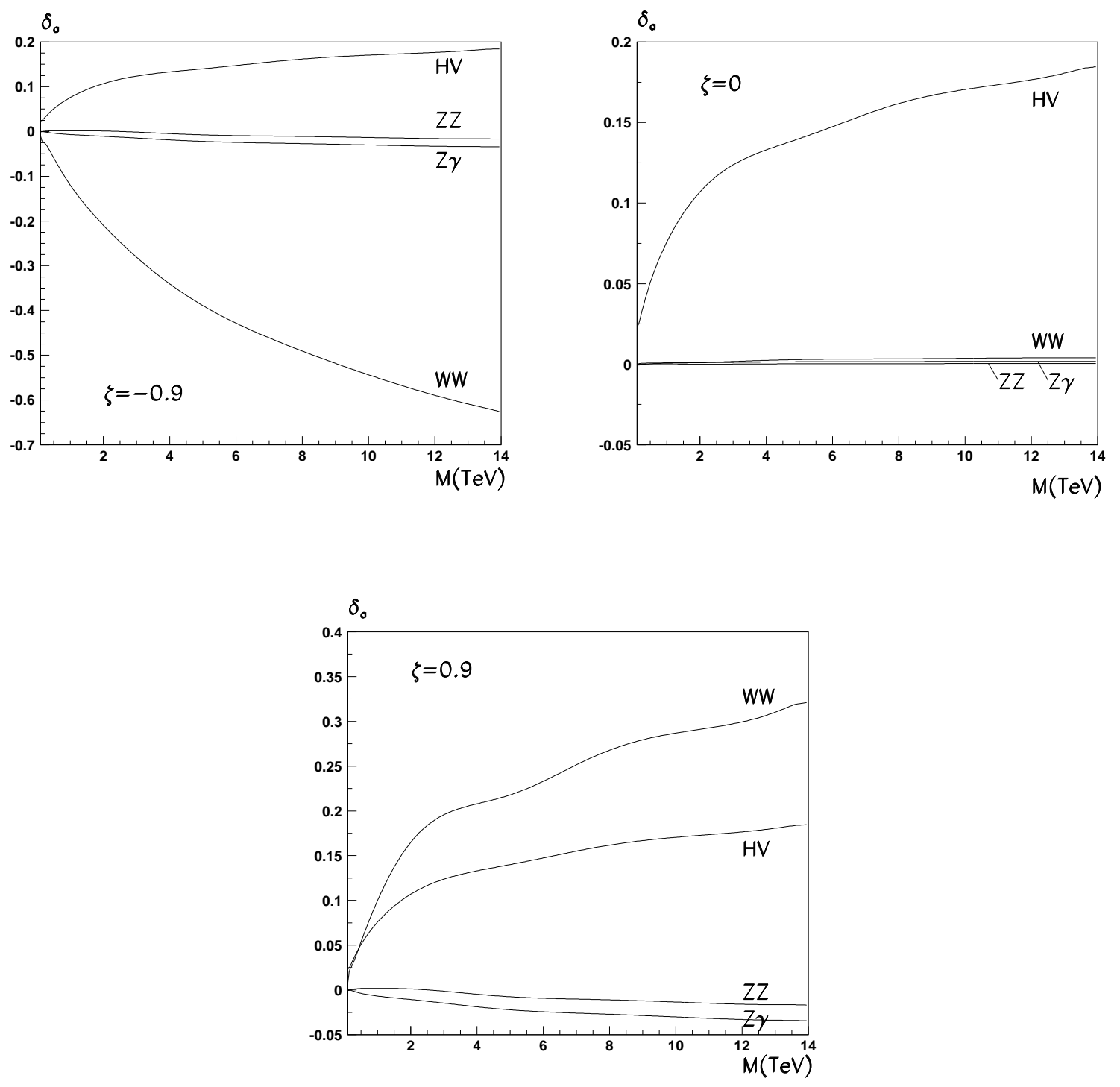

FIG. 2: The relative corrections $\delta_{a}$ corresponding to $Z Z, Z \gamma, W W, H V$-contributions for $y=0$ with different $\zeta$ as a functions of di-lepton mass $M$. 

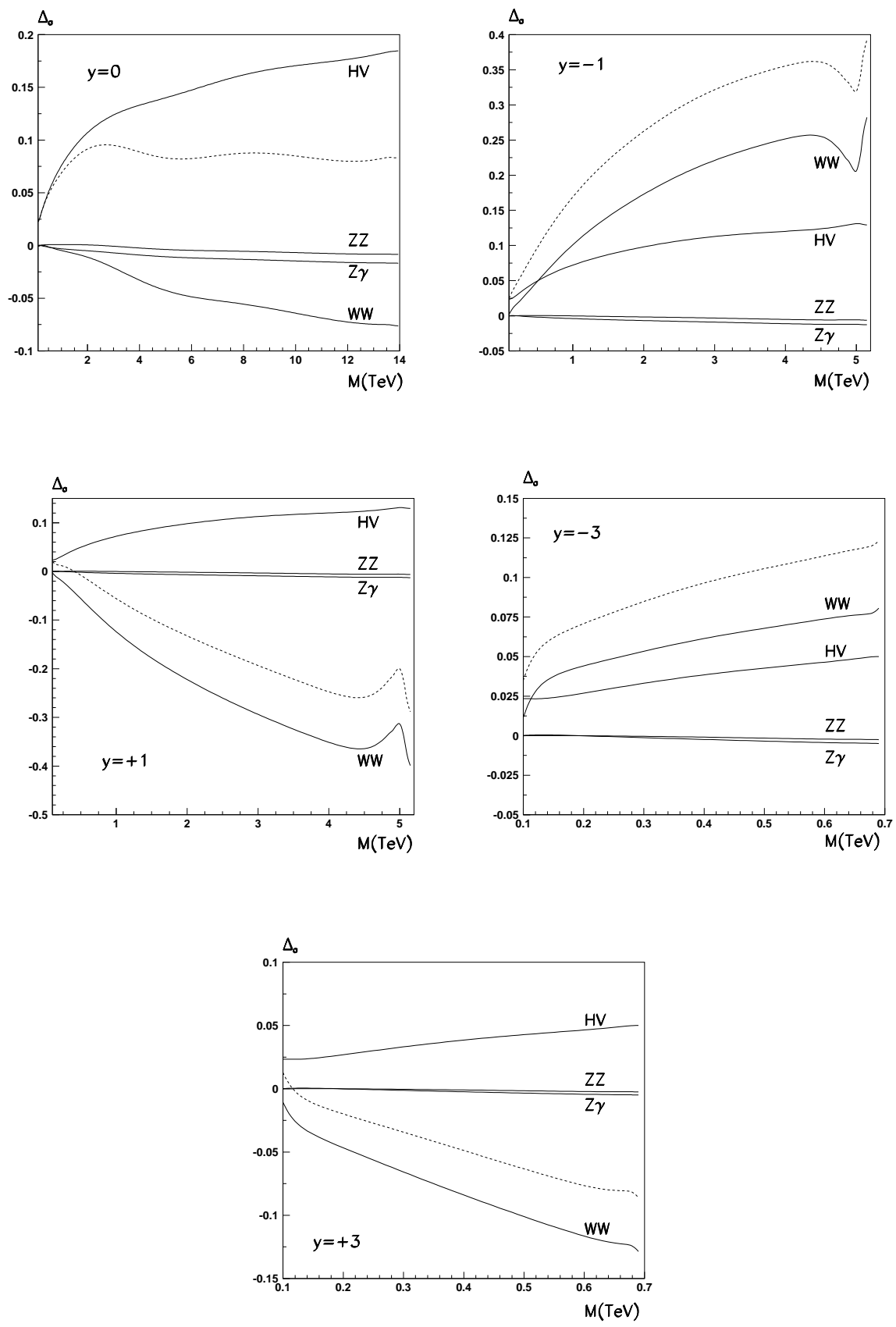

FIG. 3: The relative corrections $\Delta_{a}$ corresponding to $Z Z, Z \gamma, W W, H V$-contributions for different $y$ as a functions of di-lepton mass $M$ (solid lines). The dashed line corresponds to sum of all contributions. 


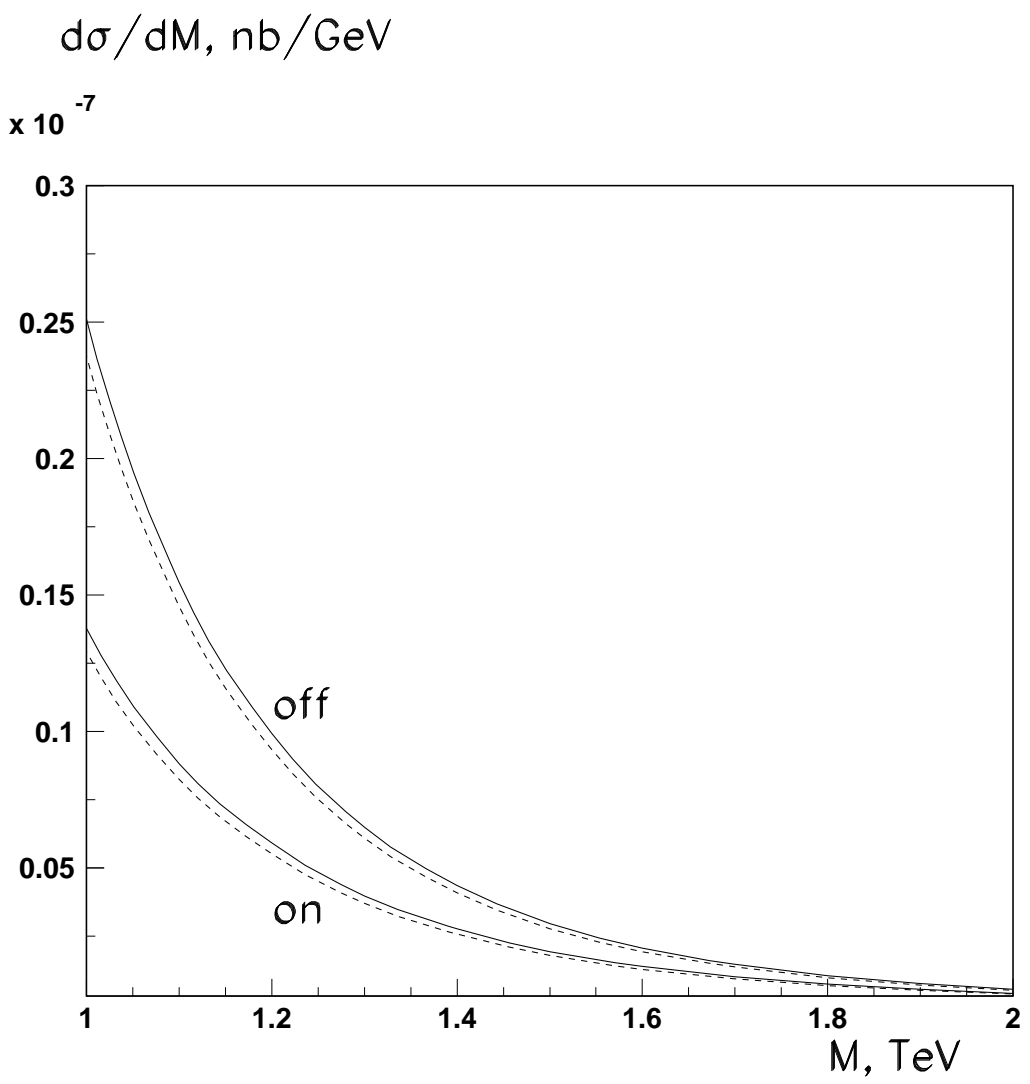

FIG. 4: The Born (dashed lines) and radiatevely corrected (solid lines) cross sections with (curves denoted by 'on') and without (curves denoted by 'off') experimental restrictions of CMS. The solid lines correspond to sum of all WRC contributions. 


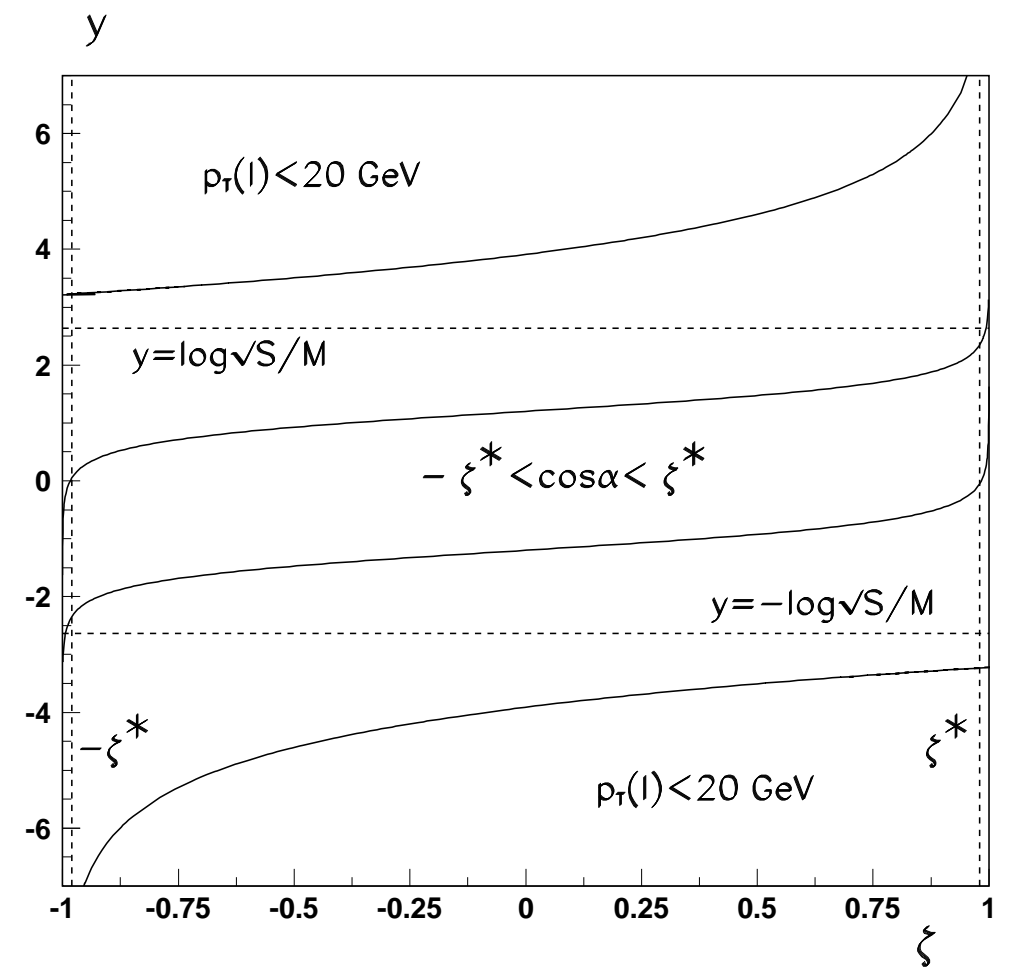

FIG. 5: The region of cross section integration over $\zeta$ and $y$ taking into consideration the experimental cuts of CMS at $\sqrt{S}=14 \mathrm{TeV}$ and $M=1 \mathrm{TeV}$. 

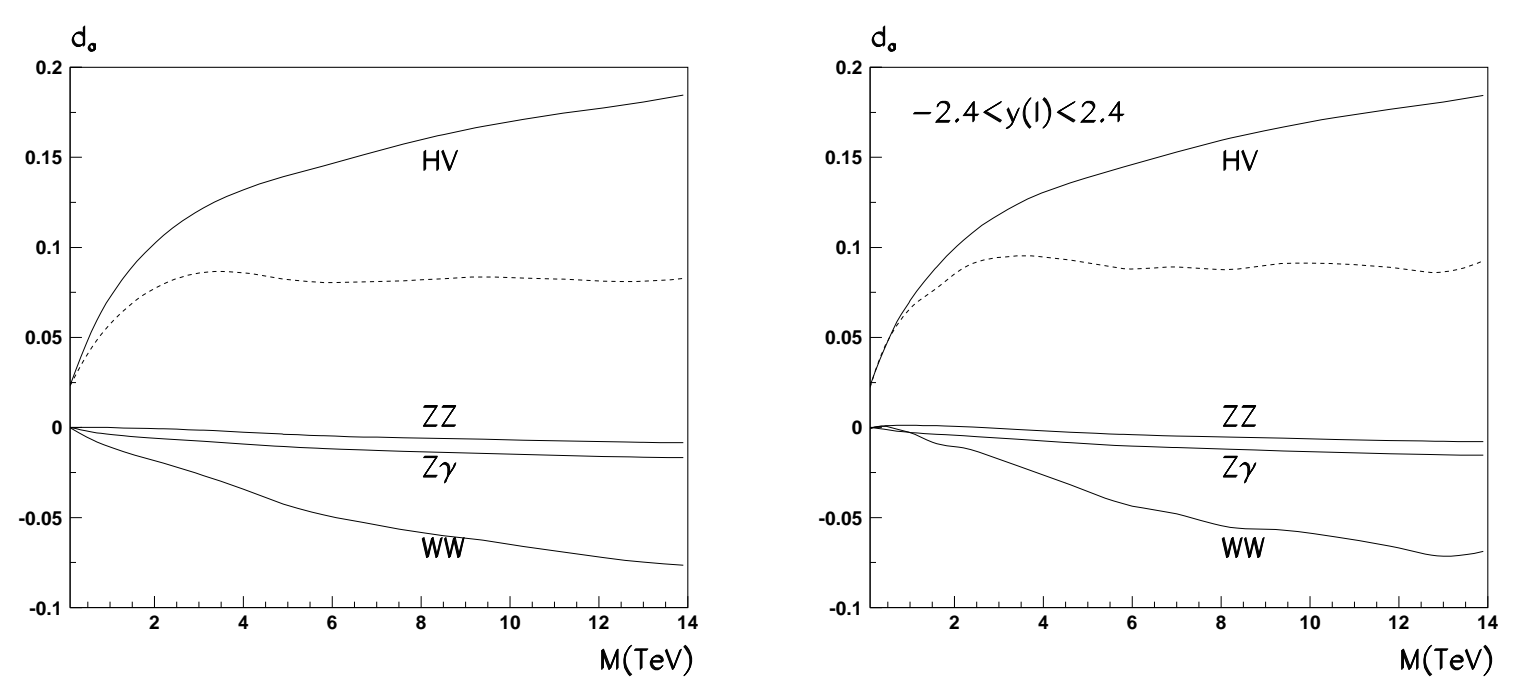

FIG. 6: The relative corrections $d_{a}$ corresponding to $Z Z, Z \gamma, W W, H V$-contributions without (left picture) and with (right picture) experimental restrictions of CMS. The dashed line corresponds to sum of all WRC contributions. 


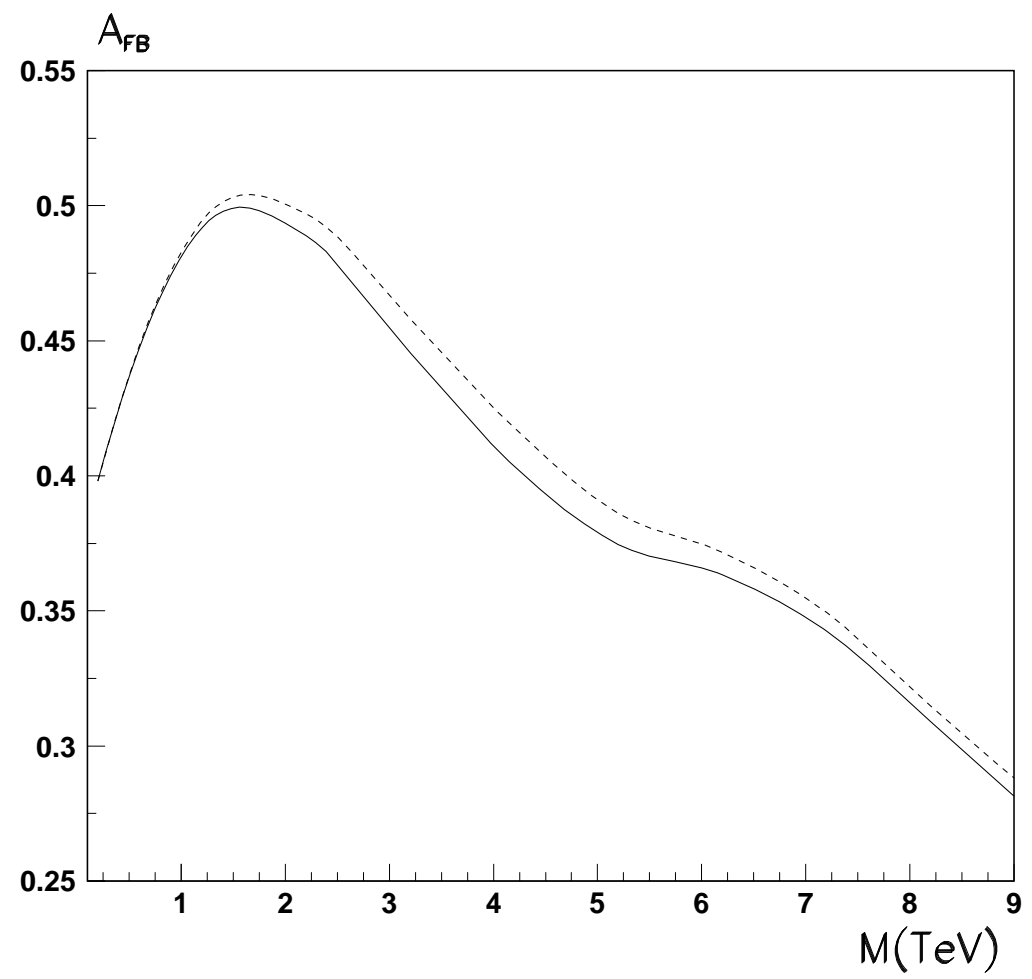

FIG. 7: The forward-backward asymmetry $A_{F B}$ in Born approximation (solid line) and taking into consideration the total WRC (dashed line) as a functions of $M$. 


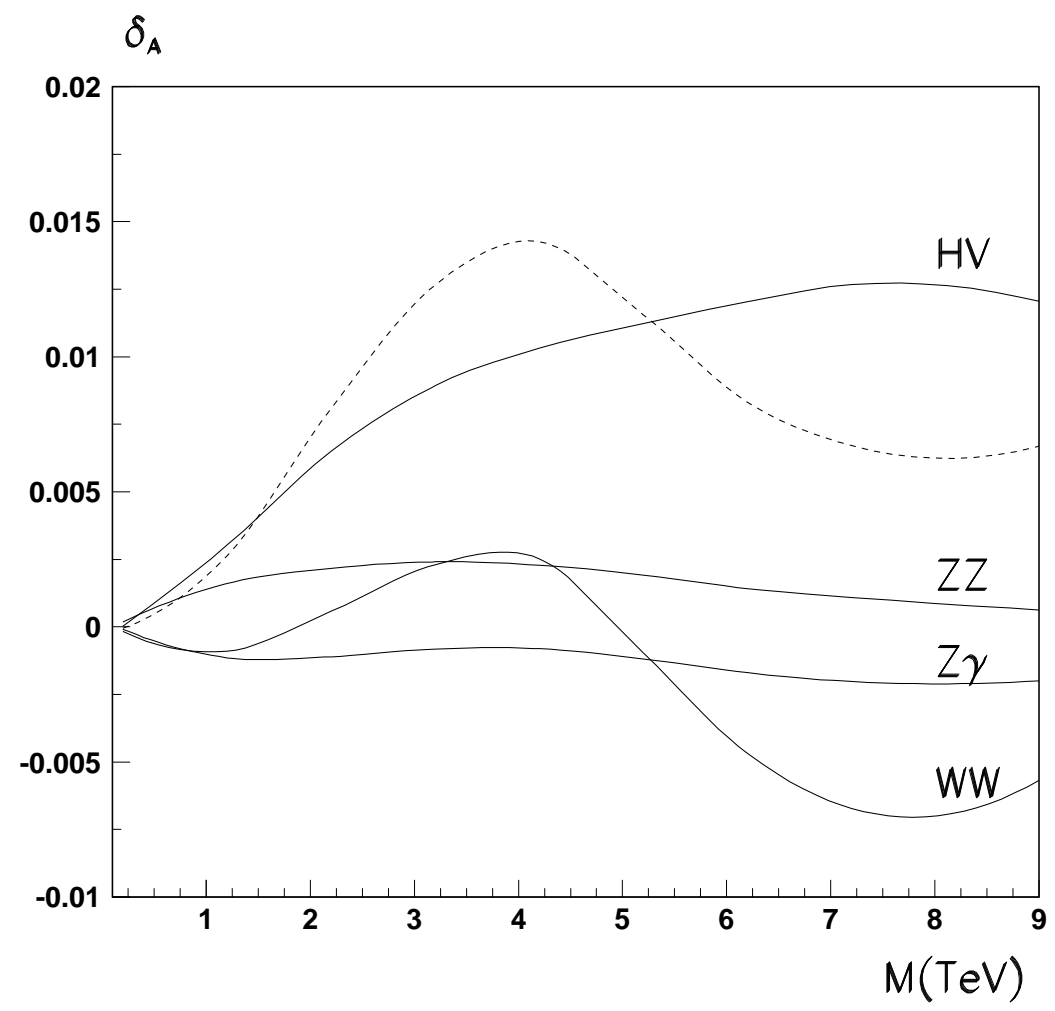

FIG. 8: The difference between Born and radiatively corrected asymmetries $\delta_{A}=A_{F B}^{a}-A_{F B}^{0}$ corresponding to $Z Z, Z \gamma, W W, H V$-contributions as a functions of $M$. The dashed line corresponds to sum of all contributions to WRC. 